\title{
BPIFB1 Inhibits Vasculogenic Mimicry Via Downregulation Of GLUT1-Mediated H3K27 Acetylation In Nasopharyngeal Carcinoma
}

\section{Xianjie Jiang}

Affiliated Cancer Hospital of School of Medicine Central South University Xiangya: Hunan Cancer Hospital

\section{Xiangying Deng}

Central South University

Jie Wang

Central South University

Yongzhen Mo

Central South University

Lei Shi

Central South University

\section{Fang Wei}

: Key Laboratory of Protein Chemistry and Developmental Biology of Ministry of Education

\section{Shanshan Zhang}

Xiangya Hospital Central South University

\section{Zhaojian Gong}

The second Xiangya Hospital,Central South University

Yi He

Affiliated Cancer Hospital of School of Medicine Central South University Xiangya: Hunan Cancer Hospital

\section{Fang Xiong}

Xiangya Hospital Central South University

\section{Yumin Wang}

Xiangya Hospital Central South University

\section{Can Guo}

Central South University

\section{Bo Xiang}

Central South University

\section{Ming Zhou}

Central South University

Qianjin Liao 
Affiliated Cancer Hospital of School of Medicine Central South University Xiangya: Hunan Cancer Hospital

\section{Xiaoling Li}

Central South University

\section{Yong Li}

Baylor College of Medicine

\section{Guiyuan Li}

Central South University

\section{Wei Xiong}

Central South University

\section{Zhaoyang Zeng ( $\nabla$ zengzhaoyang@csu.edu.cn )}

Xiangya Hospital Central South University https://orcid.org/0000-0002-0648-0565

\section{Research}

Keywords: Nasopharyngeal carcinoma, BPIFB1, GLUT1, H3K27 acetylation, Vasculogenic mimicry Posted Date: February 11th, 2021

DOl: https://doi.org/10.21203/rs.3.rs-196382/v1

License: (c) (1) This work is licensed under a Creative Commons Attribution 4.0 International License. Read Full License

Version of Record: A version of this preprint was published at Oncogene on November 1st, 2021. See the published version at https://doi.org/10.1038/s41388-021-02079-8. 


\section{Abstract}

\section{Background}

Nasopharyngeal carcinoma (NPC) exhibits significant regional differences and a high incidence in Southeast Asia and southern China. Bactericidal/permeability-increasing-fold-containing family $B$ member 1 (BPIFB1) is a relatively specific and highly expressed protein in the nasopharyngeal epithelium. Accumulating evidence indicates that BPIFB1 is substantially downregulated in NPC and low BPIFB1 is associated with NPC patient's poor prognosis. However, the clear molecular mechanism by which BPIFB1 regulates NPC is not well understood.

\section{Methods}

The expression of BPIFB1 was analyzed by immunohistochemistry $(\mathrm{IHC})$ and the vasculogenic mimicry was examined by CD31/PAS double staining in NPC clinical samples. The regulation of BPIFB1 on vasculogenic mimicry in vitro and in vivo were performed by tube formation assay and xenograft assay respectively. ECAR was analyzed by Seahorse XF analyzer. 2-NBDG uptake was detected by FACS. Glucose consumption and lactate production were analyzed by Automatic Biochemical Analyzer. Downstream targets of BPIFB1 were validated by western blot, qPCR, Chip-qRT-PCR and dual luciferase reporter assay. Lastly, immunohistochemistry $(\mathrm{IHC})$ was performed to confirm the relationship between BPIFB1 and related genes in NPC clinical samples.

\section{Results}

In this study, we show that BPIFB1 plays an important role in regulating vasculogenic mimicry. Ectopic expression of BPIFB1 significantly inhibits vasculogenic mimicry. Mechanistically, we show that BPIFB1 can inhibits JNK/AP1 signaling which leads to inhibition of glucose transporter 1 (GLUT1) transcription and glycolysis, consequently, resulting in reducing histone H3K27 acetylation and decreasing the expression of vasculogenic mimicry-related VEGFA, VE-cadherin, and MMP2.

\section{Conclusion}

This study demonstrates that BPIFB1 has a novel biological function in inhibiting glycolysis and vasculogenic mimicry and highlights that BPIFB1 is a potential target for NPC diagnosis and treatment.

\section{Background}

Nasopharyngeal carcinoma (NPC) is a malignant tumor originating from the epithelium of the nasopharynx [1]. The incidence of NPC demonstrates substantial regional variations, with a high incidence in Southeast Asia and southern China, and lower occurrence in North America, Northern Europe, and East Africa [2, 3]. Etiological studies have shown that NPC results from a combination of genetic, environmental, and dietary factors as well as EBV (Epstein-Barr virus) infection and dysregulated expression of various oncogenic and tumor suppressor proteins $[4,5]$. 
BPIFB1 (Bactericidal/permeability-increasing-fold-containing family B member 1), also known as LPLUNC1 (long palate, lung and nasal epithelium clone 1), is a member of the BPI/PLUNC superfamily as it contains two BPI domains [6]. In a previous study, we used cDNA microarrays and suppression subtractive hybridization, and demonstrated that BPIFB1 is relatively specifically to the nasopharyngeal epithelium. However, it shows low expression in NPC tissues and is negatively associated with poor prognosis in patients with NPC [7]. Our previous data showed that BPIFB1 inhibits the growth of NPC cells in an IL-6-induced inflammatory environment and their migration and invasion via vitronectin and vimentin (VIM) interactions [8-10]. However, the other biological functions of BPIFB1 in NPC remain unclear.

Tumor neovascularization through angiogenesis or vasculogenic mimicry (VM), is essential for the rapid growth and metastasis of solid tumors [11]. Angiogenesis involves the migration of endothelial cells into tumors and formation of new blood vessels $[12,13]$. However, tumor cells also can mimic endothelial cells to form tubular structures via a process called VM that was first identified in uveal melanoma and subsequently in highly aggressive tumors including glioma as well as breast, ovarian, and liver cancers $[14,15]$. However, VM and its regulatory mechanisms in NPC remain unexplored.

In this study, we investigated the role of BPIFB1 in VM by NPC cells using clinical samples, in vitro experiments, as well as a mouse model of NPC. We found that BPIFB1 inhibits VM formation through downregulation of glucose transporter 1 (GLUT) mediated H3K27 acetylation.

\section{Results}

\section{BPIFB1 inhibits VM in NPC}

In this study, 52 NPC tissues and 26 non-tumor nasopharyngeal epithelial tissues (NPE, Table S1) were collected. Immunohistochemistry results showed high BPIFB1 expression in NPE tissues, but low or even undetectable expression in NPC tissues (Fig. 1a). VM structures were examined by double staining for the endothelial cell marker CD31 and periodic acid-Schiff (PAS), a reagent that detects polysaccharides outlining the basement membrane [16]. We examined 52 NPC tissues and found that VM (yellow arrow) was more pronounced in the NPC tissues with low BPIFB1 expression but was less in cases with medium BPIFB1 expression (Fig. 1b and 1c), suggesting that BPIFB1 may play an important role in inhibiting VM formation in NPC.

To investigate the effect of BPIFB1 on VM formation in NPC, the BPIFB1 overexpression vector tagged with Flag was transfected into HONE1 and HNE2 NPC cell lines (Fig. S1). Tube formation assays were performed to investigate the effect of BPIFB1 on VM formation. The results showed that BPIFB1 significantly decreased the number of tubes in HONE1 and HNE2 cells (Fig. 1d) and downregulated the expression of VM-related vascular endothelial growth factor A (VEGFA), vascular endothelial cadherin (VE-cadherin) and matrix metalloproteinase 2 (MMP2) at the mRNA (Fig. 1e) and protein levels (Fig. 1f). These results suggest that BPIFB1 can inhibit VM formation in NPC cells. 


\section{BPIFB1 reduces VM formation by inhibiting glycolysis in NPC cells}

To investigate the possible mechanism of VM inhibition caused by BPIFB1 in NPC, signaling pathway enrichment was performed using gene set enrichment analysis of the GSE64634 NPC dataset that contains four normal nasopharyngeal tissues and 12 NPC tissues; the results showed that metabolic reprogramming was significantly dysregulated in NPC (Fig. 2A). Thus, metabolic abnormality may be one of the important factors contributing to NPC occurrence. Further analysis showed that BPIFB1 was negatively associated with several glycolysis-related genes in both GSE64634 and GSE53819 (Fig. 2b and S1b, Table S2), indicating that BPIFB1 may regulate glycolysis during the metabolic reprogramming of NPC.

To identify whether BPIFB1 affects glycolysis in NPC cells, the Seahorse XF analyzer assay was performed; the results indicated that BPIFB1 could effectively inhibit glycolysis, including basal glycolysis, maximal glycolysis, and reserve glycolysis in HONE1 and HNE2 cells (Fig. 2c). BPIFB1 could also inhibit 2-NBDG uptake, glucose consumption, and lactate production in HONE1 and HNE2 cells (Fig. $2 \mathrm{~d}$ and $2 \mathrm{e}$ ). GLUT1 is an important glucose transporter protein in cell membranes that directly affects glycolysis. qRT-PCR and western blotting experiments showed that BPIFB1 significantly downregulated GLUT1 expression at both the mRNA (Fig. S1c and S1d) and protein levels (Fig. 2f). Further, a significant negative correlation was observed between the expression of BPIFB1 and GLUT1 in the GSE53819 and GSE64634 datasets (Fig. S1e).

To further identify the role of GLUT1 in NPC glycolysis, the Seahorse XF analyzer assay was performed and showed that knockdown of GLUT1 using two siRNAs (Fig. S2a) decreased glycolysis in HONE1 and HNE2 cells, with a significant decrease in basal glycolysis, maximal glycolysis, and reserve glycolysis (Fig. S2b and S2c). GLUT1 knockdown further decreased 2-NBDG uptake, glucose consumption, and lactate production (Fig. S2d and S2e). When BPIFB1 and GLUT1 were simultaneously overexpressed in HONE1 and HNE2 cells (Fig. 2g), GLUT1 impaired the inhibitory effect of BPIFB1 on glycolysis (Fig. 2h).). Similar results were obtained in the 2-NBDG uptake assay, glucose consumption and lactate production assay (Fig. 2i and 2j). These data suggest that BPIFB1 inhibits glycolysis in NPC cells by downregulating GLUT1.

To further investigate whether BPIFB1 inhibits VM formation by downregulating GLUT1-mediated glycolysis in NPC, the tube formation assay was performed to detect the effect of GLUT1 knockdown on VM formation. The results showed that GLUT1 knockdown significantly decreased the tube formation in NPC cells as well as the expression of VEGFA, VE-cadherin, and MMP2 (Fig. 3a-3c). In contrast, GLUT1 overexpression promoted tube formation and the expression of VEGFA, VE-cadherin, and MMP2; it also attenuated the BPIFB1-induced inhibition of tube formation (Fig. 3d-3f). These data indicate that BPIFB1 could inhibit VM formation in NPC via downregulation of GLUT1-mediated glycolysis.

BPIFB1 downregulates VEGFA, VE-cadherin, and MMP2 by inhibiting GLUT1-mediated H3K27 acetylation 
Glycolysis can produce a large amount of acetyl coenzyme A, which is an important donor for histone acetylation [17-19]. To investigate whether BPIFB1 affects histone acetylation through glycolysis inhibition, we analyzed the modification of histone acetylation on VEGFA, VE-cadherin, and MMP2 using the online UCSC database. A large number of acetylated histone H3K27 enrichment signals were found on the promoters or within these three genes (Fig. S3a), suggesting that VEGFA, VE-cadherin, and MMP2 may be highly modified by histone H3K27 acetylation. The glycolysis inhibitor 2-DG could downregulate acetyl coenzyme A expression and H3K27 acetylation in HONE1 and HNE2 cells (Fig. S3b and S3c). BPIFB1 overexpression decreased intracellular acetyl coenzyme A and inhibited histone H3K27 acetylation (Fig. 4a and 4b); GLUT1 knockdown also showed similar results (Fig. S3d and S3e). GLUT1 could attenuate the downregulation of intracellular acetyl coenzyme A and H3K27 acetylation caused by BPIFB1 when both BPIFB1 and GLUT1 were overexpressed (Fig. 4c and 4d). Chromatin immunoprecipitation (ChIP)-qRT-PCR showed that BPIFB1 overexpression or GLUT1 knockdown significantly reduced the enrichment of acetylated H3K27 on VEGFA, VE-cadherin, and MMP2 (Fig. 4e and S3f). GLUT1 overexpression also attenuated the inhibited enrichment of acetylated H3K27 on VEGFA, VEcadherin, and MMP2 genes (Fig. 4f). These data suggest that BPIFB1 can inhibit histone H3K27 acetylation through GLUT1 downregulation, which reduced the enrichment of acetylated histone H3K27 on VEGFA, VE-cadherin, and MMP2, resulting in inhibition of VM formation in NPC cells.

\section{BPIFB1 downregulates GLUT1 expression by inhibiting the JNK/AP1 signaling pathway}

To investigate the molecular mechanism of BPIFB1 in regulating GLUT1, vectors were constructed for luciferase expression under GLUT1 promoters (PGL3-GLUT1-P1, containing a -2,616 bp-+112 bp fragment on GLUT1 promoter and PGL3-GLUT1-P2, containing a -312 bp +112 bp fragment on the possible core promoter region of GLUT1). Dual luciferase reporter assay showed that BPIFB1 inhibited the activities of both these reporters (Fig. 5a), suggesting that BPIFB1 regulates GLUT1 transcription mainly through the -312 bp +112 bp region of the GLUT1 promoter. ECR browser online analysis predicted the presence of NF-KB and AP1 binding sites on the -312 bp-+112 bp region of the GLUT1 promoter. Dual luciferase reporter assays revealed that BPIFB1 significantly inhibited the activity of the classical AP1 reporter and PGL3-GLUT1-P2 reporter, which contains the NF-KB and AP1 binding sites. BPIFB1 could not inhibit the activity of the PGL3-GLUT1-P2-mut reporter when the AP1 binding site of the -312 bp-112 bp region was mutated (Fig. 5b). However, mutation of the NF-KB binding site in the -312 bp-+112 bp region did not affect the regulation of this promoter reporter by BPIFB1 (data not show), suggesting that BPIFB1 regulates GLUT1 expression through the AP1 binding site on the GLUT1 promoter.

We then examined whether BPIFB1 regulates GLUT1 expression by downregulating the phosphorylation of JNK and c-Jun. Western blot experiments showed that BPIFB1 could inhibit JNK and c-Jun phosphorylation (Fig. 5c). The JNK inhibitor SP600125 inhibited the phosphorylation of JNK and c-Jun and the expression of GLUT1 (Fig. 5d); it also significantly reduced the luciferase activities of the AP1 reporter and PGL3-GLUT1-P2 reporter (Fig. S4a). The JNK activator, anisomycin, significantly increased the luciferase activities of the AP1 reporter and PGL3-GLUT1-P2 reporter and attenuated their suppression induced by BPIFB1 (Fig. S4b and S4c); further, it increased GLUT1 expression by attenuating the 
suppressed phosphorylation of JNK and c-Jun (Fig. 5e). These data indicate that BPIFB1 can regulate GLUT1 by inhibiting the JNK/AP1 signaling pathway.

\section{BPIFB1 inhibits VM formation by downregulating GLUT1 in vivo}

To investigate whether BPIFB1 could inhibit VM formation through GLUT1 in vivo, a subcutaneous NPCbearing mouse model was generated. The results showed that BPIFB1 overexpression significantly inhibited the growth of transplanted tumor cells in nude mice, whereas overexpression of GLUT1 alone promoted their growth; moreover, GLUT1 overexpression could weaken the growth inhibiting effect of BPIFB1 on the tumor cells in nude mice (Fig. 6a and 6b). CD31/PAS double staining experiments on xenograft tissues revealed that the VM were significantly fewer in the BPIFB1 overexpression group than in the control group, and that the VM was found most pronounced in the GLUT1 overexpression group; the VM were significantly more in the BPIFB1 and GLUT1 overexpression group than those in the BPIFB1 overexpression group (Fig. 6c). Immunohistochemistry results revealed that GLUT1, VEGFA, VE-cadherin, and MMP2 expression in the BPIFB1 overexpression group was significantly lower than that in the control group, whereas that in the group overexpressing both BPIFB1 and GLUT1 was higher than that in the BPIFB1 overexpressing group (Fig. 6d). These results suggest that BPIFB1 can inhibit VM formation by downregulating GLUT1 and can suppress the VM-related molecules in vivo.

\section{BPIFB1 and VM-related proteins are negatively correlated in NPC clinical samples}

Immunohistochemistry to examine the expression of VM-related molecules in clinical samples showed that BPIFB1 expression was lower in NPC tissues compared to that in NPE tissues, whereas GLUT1, VEGFA, VE-cadherin, and MMP2 were highly expressed in NPC tissues, compared with those in NPE tissues (Fig. 7a and 7b). BPIFB1 expression was negatively correlated with the expression of GLUT1, VEGFA, VE-cadherin and MMP2 (Fig. 7c and 7d), suggesting that BPIFB1 plays an important role in the inhibition of VM formation in NPC.

\section{Discussion}

In this study, we found that BPIFB1 could inhibit GLUT1 transcription through downregulation of the JNK/AP1 signaling pathway, which alters metabolic reprogramming and inhibits glycolysis in NPC cells. Inhibition of glycolysis reduces intracellular acetyl coenzyme A production, which decreases the acetylation of H3K27 and expression of VEGFA, VE-cadherin, and MMP2, ultimately leading to the inhibition of VM formation in NPC (Fig. 7e). This is an important discovery in the regulation of VM formation in NPC. Solid tumors cannot grow beyond 1-2 $\mathrm{mm}$ in diameter without neovascularization [15]. Several studies have demonstrated tumor angiogenesis in NPC, but there are very few reports on VM in NPC [20-22]. Although there are many anti-tumor drugs targeting tumor angiogenesis, their clinical results are not satisfactory [23-25]. By inhibiting vascular endothelium-derived angiogenesis alone, tumor cells can reconstruct vascular networks in tumor tissues through VM; this may be one of the reasons for the poor effect of anti-angiogenic drugs. 
Similar to angiogenesis, tumor VM aims to meet the metabolic demands of tumor cells. Multiple protumor angiogenesis signals are also known to promote tumor VM formation [26, 27]. VEGFA is an important influential factor in tumor angiogenesis, and can activate the VEGFR signaling pathway by binding to its receptor, VEGFR, to promote tumor angiogenesis [28]. VEGFA can also promote VM formation in tumors. In salivary gland adenoid cystic carcinoma, VEGFA induced by hypoxia can cause EMT in tumor cells and ultimately promote VM formation [26]. EBV infection is a clear causative factor for NPC. It was reported that EBV-encoded miR-BART1-5p can also promote glycolysis in NPC by activating the AMPK/mTOR/HIF-1 signaling axis [29]. In addition, EBV-encoded LMP1 is reported to promote VM formation via VEGFA/VEGFR1 signaling [30]. Lin Feng et al. also found that EBV-encoded LMP2A promotes VM formation through activation of PI3K/AKT/mTOR/HIF-1a signaling [16]. HIF-1a is an upstream transcription factor for key glycolysis genes such as GLUT1 and HK2 genes. This imply that EBV-encoded LMP2A may promotes VM formation in NPC by regulating intracellular metabolism. In this study, we found that the abnormal glycolysis caused by BPIFB1 overexpression could inhibit VM formation in NPC.

In this study, the abnormal glycolysis caused by BPIFB1 was found to inhibit histone acetylation. Glycolysis provides energy to tumor cells as well as regulates gene expression through various epigenetic modifications, such as histone methylation and acetylation [31-33]. It stimulates the acetyltransferase Gcn5 to acetylate H3K14; acetylated H3K14 can then inhibit Jhd2 binding to chromatin and increase H3K4 trimethylation, ultimately regulating gene expression and cell survival during aging [34]. Glycolysis can also promote T helper cell differentiation by increasing H3K27 acetylation levels in T helper cells, leading to increased expression of interferon- $\gamma$ [17].

H3K27 acetylation plays an important role in the development and treatment of tumors [35]. H3K27 acetylation can induce the expression of a variety of pro-oncogenic molecules in tumor cells. In colorectal cancer, H3K27 acetylation induces the expression of the IncRNA EIF3J-AS1, which promotes colorectal cancer cell proliferation and reduces apoptosis [36]. In oral squamous carcinoma, H3K27 acetylation promotes cell proliferation and migration by activating the IncRNA CCAT1 [37]. In breast cancer, H3K27 acetylation activates EMT and promotes trastuzumab resistance through IncRNA TINCR [38]. In this study, we found that BPIFB1 reduced acetyl coenzyme A by downregulating glycolysis, which leads to decreased acetylation of H3K27 and downregulation of VEGFA, VE-cadherin, and MMP2, resulting in the inhibition of VM formation. However, there are some shortcomings in this study. Acetyl coenzyme $A$ is an important donor of histone acetylation and their productions can alter the histone acetylation status, which regulates the expression of genes and cell biological processes. In this paper, we only examined the change of H3K27 acetylation induced by BPIFB1, but the other histone acetylation modification were not detected. Meanwhile, it is not clear whether the glycolytic inhibition induced by BPIFB1 will have an effect on the regulation of other gene expression. It is worthy for further study in the future.

Tumor VM formation involves epithelial to endothelial conversion of tumor cells, which is accompanied with the increased expression of endothelial marker molecules; for instance, tight junction proteins such as E-cadherin and Occludin in epithelial cells are downregulated, whereas endothelial cell adhesion 
molecules such as vimentin, VE-cadherin, and vitronectin are upregulated [39]. In this study, we found that VE-cadherin expression was altered by BPIFB1 during VM formation. Our previous studies also showed that BPIFB1 could reduce the expression of endothelial cell adhesion molecules, vimentin and vitronectin, and could promote epithelial marker E-cadherin expression by binding vimentin and vitronectin [10]. These indicate that BPIFB1 can inhibit the conversion of tumor cells to endothelial types. In addition, Inhibition of VM formation by BPIFB1 can also help inhibit the migration and invasion of NPC cells.

An increase in glycolysis is reported to promote radioresistance in NPC cells [40, 41]. In this study, BPIFB1 was found to downregulate glycolysis in NPC by inhibiting GLUT1. BPIFB1 enhanced the sensitivity of NPC cells to radiotherapy in our previous study. These results indicate that restoring BPIFB1 expression in NPC alone or in combination with glycolytic inhibitors, may provide new targets and strategies for the treatment of NPC.

\section{Conclusions}

In conclusion, this study revealed for the first time that BPIFB1 inhibits VM formation in NPC by altering the glycolytic process. It expands our knowledge of the regulatory role of BPIFB1 in NPC, and provides a new reference direction and theoretical basis for the treatment of NPC and for the development of antitumor angiogenesis targeting drugs.

\section{Material And Methods}

\section{Cell culture and antibodies}

NPC cell lines HONE1 and HNE2 were maintained in our laboratory. The cells were cultured in RPMI-1640 medium supplemented with $10 \%$ fetal bovine serum (Invitrogen, Shanghai, China), penicillin $(100 \mathrm{U} / \mathrm{ml})$, and streptomycin $(100 \mu \mathrm{g} / \mathrm{mL})$ in a humidified incubator under $5 \% \mathrm{CO}_{2}$ at $37^{\circ} \mathrm{C}$. The antibodies used are listed in Table S3.

\section{Constructs and reagents}

The p-IRES-Flag-BPIFB1 plasmid was constructed previously; p-CMV3-GLUT1 was purchased from Sino Biological (Beijing, China). GLUT1 siRNAs were purchased from GenePharma (Shanghai, China). The Neofect Reagent (Neofect biotech Co., Ltd. China) was used for plasmid transfection and Hiperfect (Qiagen, Hilden, Germany) was used for siRNA transfection according to the manufacturer's protocol. Reagents 2-deoxy-D-glucose(2-DG) and 2-deoxy-2-[(7-nitro-2,1,3-benzoxadiazol-4-yl)amino]-D-glucose(2NBDG) were purchased from MedChemExpresss (MCE USA); SP600125 and anisomycin were from Selleck Chemicals (Houston, TX, USA).

\section{NPC clinical samples}


In total, 26 non-tumor NPE tissues and 52 NPC samples were collected at the Second Xiangya Hospital of Central South University (Changsha, China). The study was approved by the Joint Ethics Committee of the Central South University Health Authority and informed consent was obtained from all participants. The diagnoses of all specimens were confirmed via histopathological examination.

\section{RNA isolation and quantitative real-time PCR}

Total RNA was isolated using TRIzol reagent (Invitrogen, USA) according to the manufacturer's protocol. cDNA synthesis was performed using a reverse transcription kit (Vazyme, Nanjing, China) according to the manufacturer's instructions. SYBR®Green (Vazyme) was used for qRT-PCR analysis on the MiniOpticon system (Bio-Rad, Hercules, CA, USA). After the reactions were completed, relative gene expression levels were calculated using the $2^{-\Delta \Delta C t}$ method, and $\beta$-actin was used as the endogenous control. The primer sequences used are listed in Table S4.

\section{Western blotting}

Total proteins were lysed using RIPA buffer (Beyotime Biotechnology, Shanghai, China) containing a protease/phosphatase inhibitor cocktail (Roche Applied Sciences, Mannheim, Germany), separated by 10\% SDS-PAGE, and transferred onto PVDF membranes (Millipore, Billerica, MA, USA). The membrane was blocked with $5 \%$ non-fat milk with TBST for 1 hour at room temperature and incubated with the primary antibodies overnight at $4{ }^{\circ} \mathrm{C}$. After washing, the membrane was incubated with HRP-labeled secondary antibodies (CUSBIO, Wuhan, China) for $1 \mathrm{~h}$ at room temperature. The proteins were then detected using ECL reagent (Millipore).

\section{Measurement of glucose uptake, lactate production, and 2-NBDG uptake}

Cells were seeded in 12-well plates $\left(10^{5} /\right.$ well); after incubation for $8 \mathrm{~h}$, the medium was replaced with fresh complete medium, and the cells were incubated for another $24 \mathrm{~h}$. Glucose and lactate levels were measured using the Automatic Biochemical Analyzer (AU680, Beckman Coulter International, Brea, CA, USA). The relative glucose consumption rate and lactate production rate were normalized to the cell numbers in the samples.

For the 2-NBDG uptake assay, cells were treated with fresh glucose-free medium containing $100 \mu \mathrm{M} 2$ NBDG for 45 min and the mean fluorescence intensity was immediately measured using flow cytometry.

\section{Glycolysis assay}

For the glycolysis assay, a Glycolysis Stress Test Kit (Agilent, USA) was used to measure the extracellular acidification rate according to the manufacturer's instructions and protocols (Seahorse Bioscience, North Billerica, MA, USA).

\section{Tube formation assay}


For the tube formation assay, $50 \mu$ of Matrigel (BD) was plated in 96-well plates and incubated at $37^{\circ} \mathrm{C}$ for $1 \mathrm{~h}$ to allow Matrigel polymerization. Then, $3 \times 10^{4}$ cells per well were seeded onto the Matrigel layer and incubated at $37^{\circ} \mathrm{C}$ for $4 \mathrm{~h}$. Randomized fields were captured using microscope. The numbers of capillary-like structures were quantified using ImageJ software. The data are presented as the average numbers of tubes \pm standard deviation (SD).

\section{Luciferase reporter assay}

All luciferase reporter constructs were generated from the PGL3-basic vector (Promega, Madison, WI, USA). The wild type and mutant sequences of GLUT1 promoter, named PGL3-GLUT1-WT and PGL3GLUT1-mut, were amplified and cloned into the PGL3-basic vector. The primers used are shown in Table S4. Luciferase activity was measured using the Dual-Luciferase Reporter Assay System (Promega). The relative luciferase signal was presented as firefly luciferase activity normalized to the Renilla luciferase activity. The AP1-luc reporter was purchased from Beyotime Biotechnology (Beijing, China).

\section{ChIP}

Cells were fixed with $1 \%$ formaldehyde for $10 \mathrm{~min}$ at room temperature, and the fixation was stopped with $0.125 \mathrm{M}$ glycine; the cell lysis buffer was then added and the samples were sonicated to generate 2001000 bp fragments. The resulting cell lysates were immunoprecipitated using H3K27 antibody (active motif, USA) and analyzed via qRT-PCR. The primers used are shown in Table S4.

\section{Animal experiments}

Female 4-week-old, nu/nu-BALB/c athymic nude mice were randomly divided into four groups (five mice each). First, $5 \times 10^{6}$ HONE1 cells transfected or co-transfected with the empty vector, BPIFB1 overexpression vector, or GLUT1 overexpression vector were injected subcutaneously into respective mice. Tumor growth was monitored every 3 days. Tumor size was assessed by measuring the largest perpendicular diameters, and the tumor volume was calculated as follows: $V=1 / 2 \times$ (length) $\times($ width $) \times$ (width). After 33 days of subcutaneous inoculation, the mice were euthanized by cervical dislocation and the tumor tissue was excised. The formed tumor masses were removed and weighed. All animal protocols were approved by the Institutional Laboratory of Animal Care and Use Committee at Central South University(2020syaw0896).

\section{Immunohistochemistry and CD31/PAS staining}

Immunohistochemistry was performed on formalin-fixed paraffin-embedded sections of clinical NPC tissues and mouse xenograft tissues. Briefly, the tissues were deparaffinized and rehydrated, and the samples were subjected to EDTA-mediated high-temperature antigen retrieval; the samples were then incubated overnight at $4{ }^{\circ} \mathrm{C}$ with the primary antibodies. The staining was scored according to the staining intensity and the distribution of stained cells. Distribution was evaluated as none (0), $\leq 10 \%(1)$, $10 \%-50 \%(2), 50 \%-80 \%(3)$, and $>80 \%$ (4). Intensity was evaluated as none (0), faint (1), moderate (2), 
strong (3), or very strong (4). The sections were reviewed by two pathologists. The final staining scores were calculated as the product of staining intensity multiplied by the percentage of stained cells[42]. To detect VM, a CD31/PAS staining Kit (Solarbio, Beijing, China) was used according to the manufacturer's instructions. The VM structure criteria were negative for CD31 but positive for PAS (CD31\% $/$ PAS $^{+}$). The numbers of positive cells were counted from $\geq 4$ randomly chosen fields.

\section{Statistical analysis}

Statistical analyses were performed using GraphPad Prism 7.0 software. Differences between groups were analyzed using the Student's t-test when there were only two groups, or using one-way ANOVA when there were more than two groups. Pearson's correlation coefficient was used to determine the correlations of BPIFB1 with GLUT1, VEGFA, VE-cadherin, and MMP2. A two-tailed value of $p<0.05$ was considered statistically significant $(*, p<0.05 ; * \star, p<0.01 ; * \star \star, p<0.001 ; * \star \star \star, p<0.0001)$.

\section{Abbreviations}

BPIFB1: Bactericidal/permeability-increasing-fold-containing family B member 1

NPC: nasopharyngeal carcinoma

NPE: non-tumor nasopharyngeal epithelial

VM: vasculogenic mimicry

GLUT1: glucose transporter 1

VEGFA: vascular endothelial growth factor $A$

VE-cadherin: vascular endothelial cadherin

MMP2: matrix metalloproteinase 2

qRT-PCR: quantitative real-time polymerase chain reaction

Chip: Chromatin immunoprecipitation

\section{Declarations}

\section{Ethics approval and consent to participate}

Written informed consent for the biological studies was obtained from each patient involved in the study, and the ethical and legal standards approved by the Research Ethics Committee of the Second Xiangya Hospital. All animal studies were approved by the Ethics Committee of the Central South University. 


\section{Consent for publication}

All authors agree to submit for consideration for publication in the journal.

\section{Availability of data and material}

The information of samples is provided in Supplementary Table 1. The data of heatmaps are provided in Supplementary Table 2, GSE64624 and GSE53819 are available in GEO datasets. The prediction of H3K27AC enrichment in VEGFA, VE-cadherin and MMP2 are available in UCSC database.

\section{Competing interests}

The authors declare that they have no conflict of interest.

\section{Funding}

This study was supported by grants from the National Natural Science Foundation of China (81903138, 81972776, U20A20367), the Overseas Expertise Introduction Project for Discipline Innovation (111 Project, No. 111-2-12), the Natural Science Foundation of Hunan Province (2019JJ50778, 2019JJ50872, 2020JJ4766)

\section{Acknowledgements}

Not applicable.

\section{Authors' information}

Affiliations

NHC Key Laboratory of Carcinogenesis, Hunan Cancer Hospital and the Affiliated Cancer Hospital of Xiangya School of Medicine, Central South University, Changsha, Hunan, China;

Xianjie Jiang,Yi He,Bo Xiang,Ming Zhou,Qianjin Liao,Xiaoling Li,Guiyuan Li,Wei Xiong\&Zhaoyang Zeng The Key Laboratory of Carcinogenesis and Cancer Invasion of the Chinese Ministry of Education, Cancer Research Institute and School of Basic Medicine Sciences, Central South University, Changsha, Hunan, China 
Xianjie Jiang,Xiangying Deng,Jie Wang,Yongzhen Mo,Lei Shi,Fang Wei,Yumin Wang,Can Guo,Bo Xiang,Ming Zhou,Xiaoling Li,Guiyuan Li,Wei Xiong\&Zhaoyang Zeng

Department of Stomatology, Xiangya Hospital, Central South University, Changsha, Hunan, China; Shanshan Zhang\&Fang Xiong

Department of Oral and Maxillofacial Surgery, The Second Xiangya Hospital, Central South University, Changsha, Hunan, China

Zhaojian Gong

Center for Aging Biomedicine, Key Laboratory of Protein Chemistry and Developmental Biology of Ministry of Education, College of Life Sciences, Hunan Normal University, Changsha, Hunan, China

Fang Wei

Department of Medicine, Dan L Duncan Comprehensive Cancer Center, Baylor College of Medicine, Houston, Texas, USA

Yong Li

\section{Authors' contributions}

XJ. Jiang performed all Experiments; ZY. Zeng designed this study; XY. Deng, J. Wang, YZ. Mo and L. Shi collected tissue samples and the clinical data; F. Wei, SS. Zhang, ZJ, Gong and Y. He gave guidance on experimental methods; F. Xiong, YM. Wang, C. Guo, B Xiang, M. Zhou, QJ. Liao, XL. Li, GY. Li, W. Xiong analyzed and interpreted the data; XJ. Jiang and ZY. Zeng drafted the manuscript. All authors read and approved the final manuscript.

Corresponding authors

Correspondence to Zhaoyang Zeng

\section{References}

1. Chua MLK, Wee JTS, Hui EP, Chan ATC. Nasopharyngeal carcinoma. Lancet. 2016;387:1012-24.

2. Wei F, Wu Y, Tang L, Xiong F, Guo C, Li X, et al. Trend analysis of cancer incidence and mortality in China. Sci China Life Sci. 2017;60:1271-75.

3. Chen YP, Chan ATC, Le QT, Blanchard P, Sun Y, Ma J. Nasopharyngeal carcinoma. Lancet. 2019;394:64-80.

4. Zeng Z, Huang H, Huang L, Sun $M$, Yan Q, Song Y, et al. Regulation network and expression profiles of Epstein-Barr virus-encoded microRNAs and their potential target host genes in nasopharyngeal carcinomas. Sci China Life Sci. 2014;57:315-26. 
5. Zeng Z, Huang H, Zhang W, Xiang B, Zhou M, Zhou Y, et al. Nasopharyngeal carcinoma: advances in genomics and molecular genetics. Sci China Life Sci. 2011;54:966-75.

6. Zhou Y, Liao Q, Li X, Wang H, Wei F, Chen J, et al. HYOU1, Regulated by LPLUNC1, Is Up-Regulated in Nasopharyngeal Carcinoma and Associated with Poor Prognosis. J Cancer. 2016;7:367-76.

7. Zhang B, Nie X, Xiao B, Xiang J, Shen S, Gong J, et al. Identification of tissue-specific genes in nasopharyngeal epithelial tissue and differentially expressed genes in nasopharyngeal carcinoma by suppression subtractive hybridization and cDNA microarray. Genes Chromosomes Cancer. 2003;38:80-90.

8. Liao Q, Zeng Z, Guo X, Li X, Wei F, Zhang W, et al. LPLUNC1 suppresses IL-6-induced nasopharyngeal carcinoma cell proliferation via inhibiting the Stat3 activation. Oncogene. 2014;33:2098-109.

9. Wang H, Zhou Y, Oyang L, Han Y, Xia L, Lin J, et al. LPLUNC1 stabilises PHB1 by counteracting TRIM21-mediated ubiquitination to inhibit NF-kappaB activity in nasopharyngeal carcinoma. Oncogene. 2019;38:5062-75.

10. Wei F, Wu Y, Tang L, He Y, Shi L, Xiong F, et al. BPIFB1 (LPLUNC1) inhibits migration and invasion of nasopharyngeal carcinoma by interacting with VTN and VIM. Br J Cancer. 2018;118:233-47.

11. Lugano R, Ramachandran M, Dimberg A. Tumor angiogenesis: causes, consequences, challenges and opportunities. Cell Mol Life Sci. 2019;

12. Weidner N, Semple JP, Welch WR, Folkman J. Tumor angiogenesis and metastasis--correlation in invasive breast carcinoma. N Engl J Med. 1991;324:1-8.

13. Folkman J. Proceedings: Tumor angiogenesis factor. Cancer Res. 1974;34:2109-13.

14. Krishna Priya S, Nagare RP, Sneha VS, Sidhanth C, Bindhya S, Manasa P, et al. Tumour angiogenesisOrigin of blood vessels. Int J Cancer. 2016;139:729-35.

15. Jiang X, Wang J, Deng X, Xiong F, Zhang S, Gong Z, et al. The role of microenvironment in tumor angiogenesis. J Exp Clin Cancer Res. 2020;39:204.

16. Xiang T, Lin YX, Ma W, Zhang HJ, Chen KM, He GP, et al. Vasculogenic mimicry formation in EBVassociated epithelial malignancies. Nat Commun. 2018;9:5009.

17. Peng M, Yin N, Chhangawala S, Xu K, Leslie CS, Li MO. Aerobic glycolysis promotes T helper 1 cell differentiation through an epigenetic mechanism. Science. 2016;354:481-84.

18. Moussaieff A, Rouleau M, Kitsberg D, Cohen M, Levy G, Barasch D, et al. Glycolysis-mediated changes in acetyl-CoA and histone acetylation control the early differentiation of embryonic stem cells. Cell Metab. 2015;21:392-402.

19. Shi L, Tu BP. Acetyl-CoA and the regulation of metabolism: mechanisms and consequences. Curr Opin Cell Biol. 2015;33:125-31.

20. Bao L, You B, Shi S, Shan Y, Zhang Q, Yue H, et al. Metastasis-associated miR-23a from nasopharyngeal carcinoma-derived exosomes mediates angiogenesis by repressing a novel target gene TSGA10. Oncogene. 2018;37:2873-89. 
21. Ma W, Feng L, Zhang S, Zhang H, Zhang X, Qi X, et al. Induction of chemokine (C-C motif) ligand 5 by Epstein-Barr virus infection enhances tumor angiogenesis in nasopharyngeal carcinoma. Cancer Sci. 2018;109:1710-22.

22. Chen S, Lv L, Zhan Z, Wang X, You Z, Luo X, et al. Silencing of long noncoding RNA SRRM2-AS exerts suppressive effects on angiogenesis in nasopharyngeal carcinoma via activating MYLK-mediated cGMP-PKG signaling pathway. J Cell Physiol. 2020;235:7757-68.

23. Mahfouz N, Tahtouh R, Alaaeddine N, El Hajj J, Sarkis R, Hachem R, et al. Gastrointestinal cancer cells treatment with bevacizumab activates a VEGF autoregulatory mechanism involving telomerase catalytic subunit hTERT via PI3K-AKT, HIF-1 alpha and VEGF receptors. PLoS One. 2017;12:e0179202.

24. Zarrin B, Zarifi F, Vaseghi G, Javanmard SH. Acquired tumor resistance to antiangiogenic therapy: Mechanisms at a glance. J Res Med Sci. 2017;22:117.

25. Loges S, Schmidt T, Carmeliet P. Mechanisms of resistance to anti-angiogenic therapy and development of third-generation anti-angiogenic drug candidates. Genes Cancer. 2010;1:12-25.

26. Wang HF, Wang SS, Zheng M, Dai LL, Wang K, Gao XL, et al. Hypoxia promotes vasculogenic mimicry formation by vascular endothelial growth factor $A$ mediating epithelial-mesenchymal transition in salivary adenoid cystic carcinoma. Cell Prolif. 2019;52:e12600.

27. Zhu Y, Liu X, Zhao P, Zhao H, Gao W, Wang L. Celastrol Suppresses Glioma Vasculogenic Mimicry Formation and Angiogenesis by Blocking the PI3K/Akt/mTOR Signaling Pathway. Front Pharmacol. 2020;11:25.

28. Claesson-Welsh L, Welsh M. VEGFA and tumour angiogenesis. J Intern Med. 2013;273:114-27.

29. Lyu X, Wang J, Guo X, Wu G, Jiao Y, Faleti OD, et al. EBV-miR-BART1-5P activates AMPK/mTOR/HIF1 pathway via a PTEN independent manner to promote glycolysis and angiogenesis in nasopharyngeal carcinoma. PLoS Pathog. 2018;14:e1007484.

30. Xu S, Bai J, Zhuan Z, Li B, Zhang Z, Wu X, et al. EBVLMP1 is involved in vasculogenic mimicry formation via VEGFA/VEGFR1 signaling in nasopharyngeal carcinoma. Oncol Rep. 2018;40:377-84.

31. Wellen KE, Hatzivassiliou G, Sachdeva UM, Bui TV, Cross JR, Thompson CB. ATP-citrate lyase links cellular metabolism to histone acetylation. Science. 2009;324:1076-80.

32. Kaelin WG, Jr., McKnight SL. Influence of metabolism on epigenetics and disease. Cell. 2013;153:5669.

33. Sabari BR, Zhang D, Allis CD, Zhao Y. Metabolic regulation of gene expression through histone acylations. Nat Rev Mol Cell Biol. 2017;18:90-101.

34. Wu Y, Zhang S, Gong X, Yu Q, Zhang Y, Luo M, et al. Glycolysis regulates gene expression by promoting the crosstalk between H3K4 trimethylation and H3K14 acetylation in Saccharomyces cerevisiae. J Genet Genomics. 2019;46:561-74.

35. Krug B, De Jay N, Harutyunyan AS, Deshmukh S, Marchione DM, Guilhamon P, et al. Pervasive H3K27 Acetylation Leads to ERV Expression and a Therapeutic Vulnerability in H3K27M Gliomas. Cancer Cell. 2019;35:782-97 e8. 
36. Liu D, Zhang H, Cong J, Cui M, Ma M, Zhang F, et al. H3K27 acetylation-induced IncRNA EIF3J-AS1 improved proliferation and impeded apoptosis of colorectal cancer through miR-3163/YAP1 axis. J Cell Biochem. 2020;121:1923-33.

37. Zhang E, Han L, Yin D, He X, Hong L, Si X, et al. H3K27 acetylation activated-long non-coding RNA CCAT1 affects cell proliferation and migration by regulating SPRY4 and HOXB13 expression in esophageal squamous cell carcinoma. Nucleic Acids Res. 2017;45:3086-101.

38. Dong H, Hu J, Zou K, Ye M, Chen Y, Wu C, et al. Activation of LncRNA TINCR by H3K27 acetylation promotes Trastuzumab resistance and epithelial-mesenchymal transition by targeting MicroRNA125b in breast Cancer. Mol Cancer. 2019;18:3.

39. Wei X, Chen Y, Jiang X, Peng M, Liu Y, Mo Y, et al. Mechanisms of vasculogenic mimicry in hypoxic tumor microenvironments. Mol Cancer. 2021;20:7.

40. Tang L, Wei F, Wu Y, He Y, Shi L, Xiong F, et al. Role of metabolism in cancer cell radioresistance and radiosensitization methods. J Exp Clin Cancer Res. 2018;37:87.

41. Lu J, Tang M, Li H, Xu Z, Weng X, Li J, et al. EBV-LMP1 suppresses the DNA damage response through DNA-PK/AMPK signaling to promote radioresistance in nasopharyngeal carcinoma. Cancer Lett. 2016;380:191-200.

42. Ju S, Wang F, Wang Y, Ju S. CSN8 is a key regulator in hypoxia-induced epithelial-mesenchymal transition and dormancy of colorectal cancer cells. Mol Cancer. 2020;19:168.

\section{Figures}




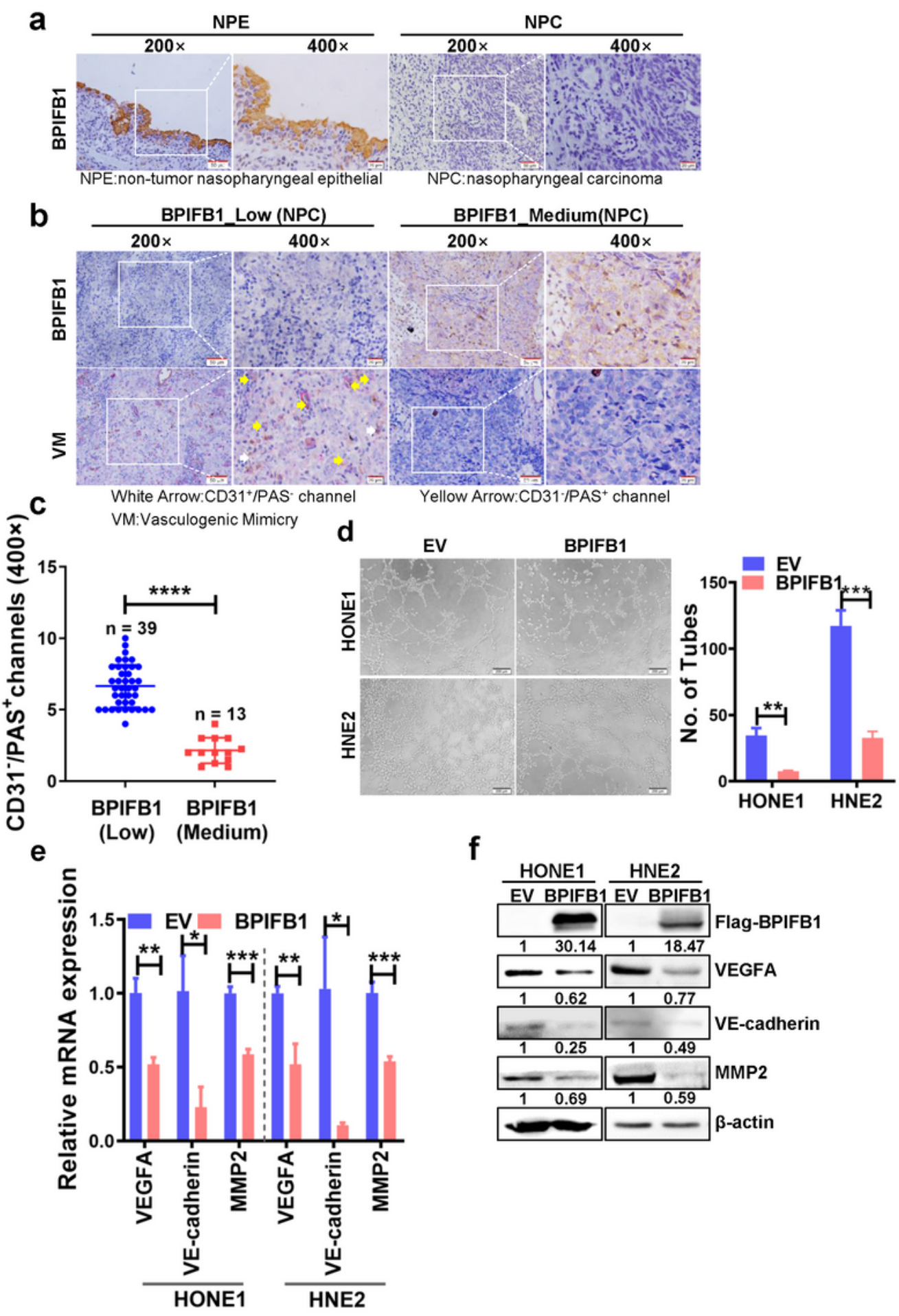

\section{Figure 1}

BPIFB1 inhibits vasculogenic mimicry in nasopharyngeal carcinoma. a. Representative results showing the BPIFB1 expression in immunohistochemically analyses in 52 NPC tissues and 26 NPE tissues. Magnification $=\times 200$, Scale bar $=50 \mu \mathrm{m}$; Magnification $=\times 400$, Scale bar $=20 \mu \mathrm{m} . \mathrm{b}$ CD31/PAS double staining for VM in 52 NPC tissues. Magnification $=\times 200$, Scale bar $=50 \mu \mathrm{m}$; Magnification $=\times 400$, Scale bar $=20 \mu \mathrm{m}$. c. The numbers of VM are inversely correlated with BPIFB1 expression in 52 NPC tissues ( $p$ 
$<0.0001)$. $d$. The tube formation assay showed that BPIFB1 overexpression inhibited tube formation by HONE1 and HNE2 cells. e. qRT-PCR showed that BPIFB1 overexpression decreased the expression of VEGFA, VE-cadherin, and MMP2 genes in HONE1 and HNE2 cells. f. Western blotting showed that BPIFB1 overexpression decreased the expression of VEGFA, VE-cadherin and MMP2 proteins in HONE1 and HNE2 cells. Data shown are representative images or expressed as the mean \pm s.d. of each group from three separate experiments (in vitro ) or one separate experiment (in vivo). ( ${ }^{\star}, p<0.05 \rrbracket * \star, p<0.01$; ${ }^{\star \star *}, p<$ $0.001 ; * \star * *, p<0.0001)$

a

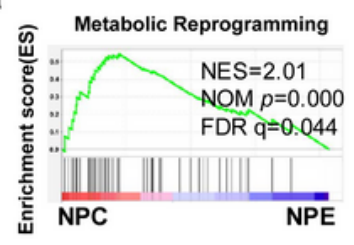

b

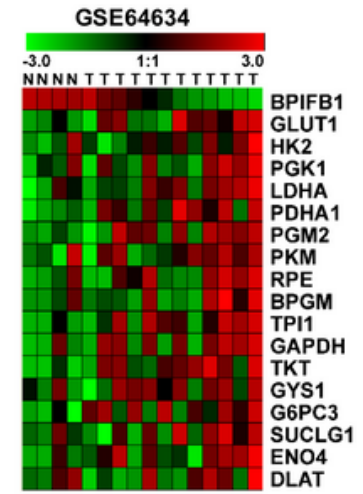

f
C
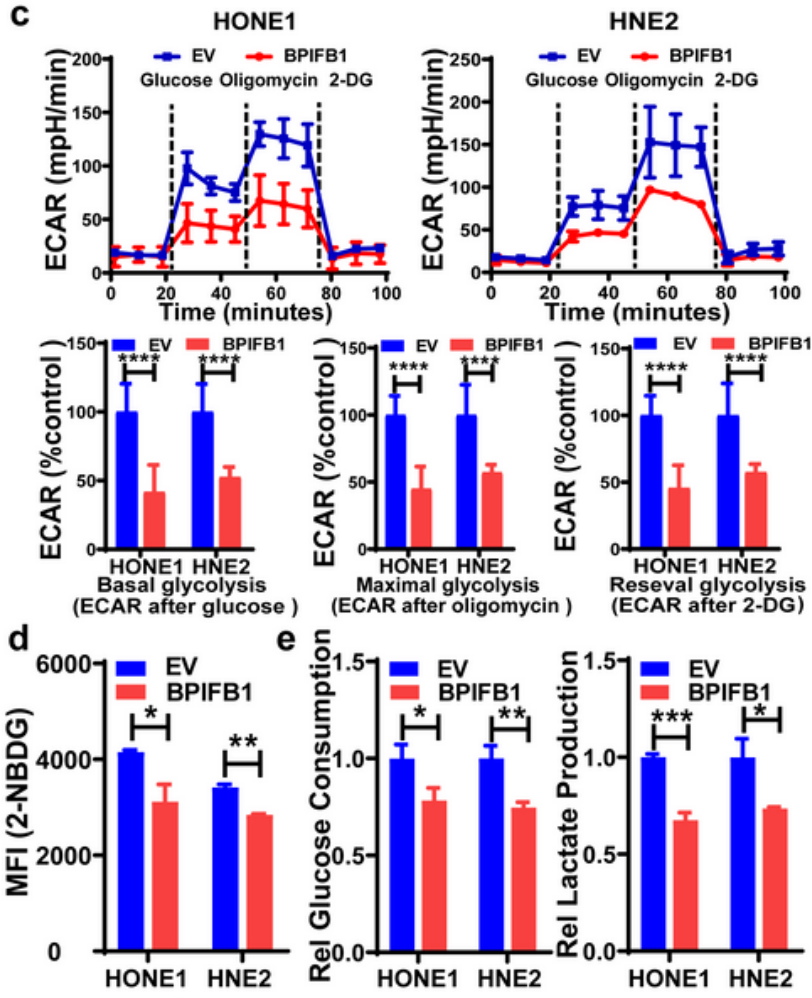

e 흐

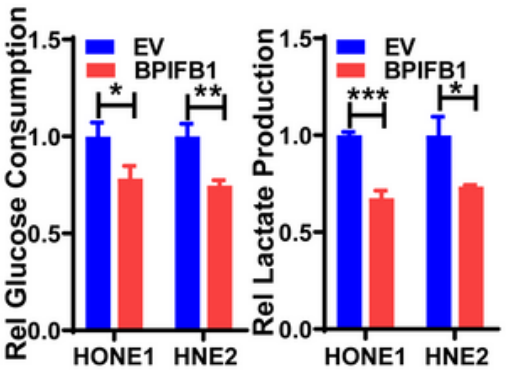

g

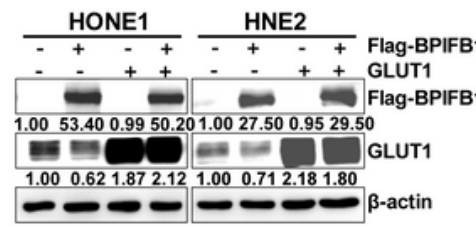

HNE2

h
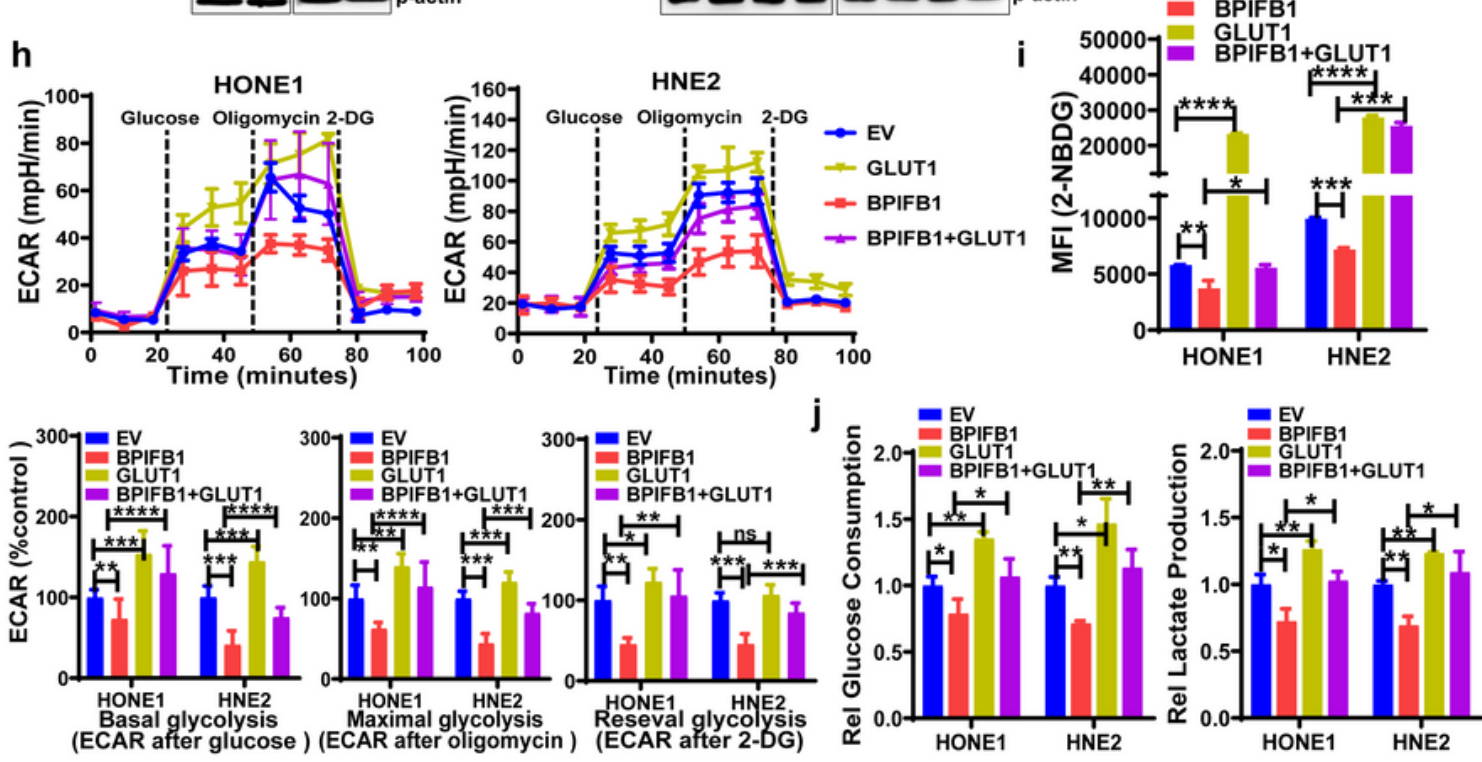


\section{Figure 2}

BPIFB1 inhibits glycolysis by downregulating GLUT1. a. Gene set enrichment analysis showed the enhanced metabolic reprogramming pathway in the GSE64634 dataset. NES: normalized enrichment score, NOM p: normalized p-value. b. BPIFB1 was inversely correlated with several glycolysis-related genes after SAM analysis of the GSE64634 dataset. c. The extracellular acidification rate (ECAR) in HONE1 and HNE2 cells after BPIFB1 overexpression, including basal glycolysis, maximal glycolysis, and reserve glycolysis was measured using Seahorse XF assays. d. The 2-NBDG uptake in HONE1 and HNE2 cells transfected with the BPIFB1 overexpression plasmid was measured via FACS as mean fluorescence intensity. e. Glucose consumption and lactate production in HONE1 and HNE2 cells transfected with the BPIFB1 overexpression plasmid were examined using an automated biochemical analyzer. $f$. Western blotting showed that BPIFB1 significantly downregulated GLUT1 protein expression in HONE1 and HNE2 cells. g. Western blotting showed the BPIFB1 and GLUT1 expression in HONE1 and HNE2 cells after cotransfection with BPIFB1 and GLUT1 overexpression plasmids. h. The extracellular acidification rate (ECAR) in HONE1 and HNE2 cells after co-transfection of BPIFB1 and GLUT1 overexpression plasmids was measured using Seahorse XF assays. i. The 2-NBDG uptake assay in HONE1 and HNE2 cells after co-transfecting the BPIFB1 and GLUT1 overexpression plasmids was performed by FACS to determine mean fluorescence intensity. j. Glucose consumption and lactate production in HONE1 and HNE2 cells after co-transfecting the BPIFB1 and GLUT1 overexpression plasmids, were examined using an automated biochemical analyzer. Data shown are representative images or expressed as the mean \pm s.d. of each group from three separate experiments or one separated experiment (microarray analysis). ( ${ }^{*}, p<$ $0.05 ; * \star, p<0.01 ; * \star *, p<0.001 ; * \star \star *, p<0.0001$ vs. control, Student's t-test) 


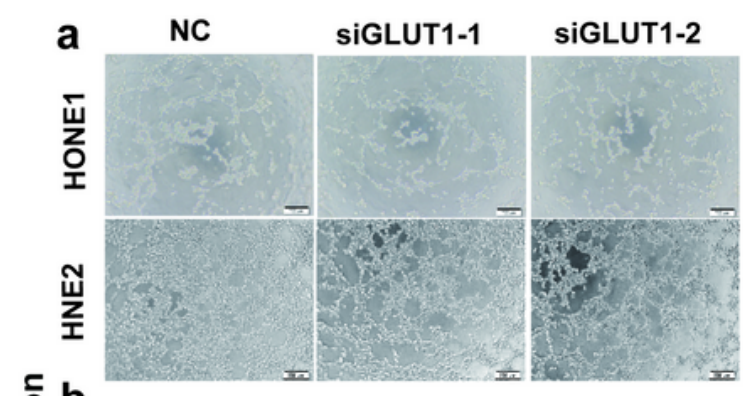

흠
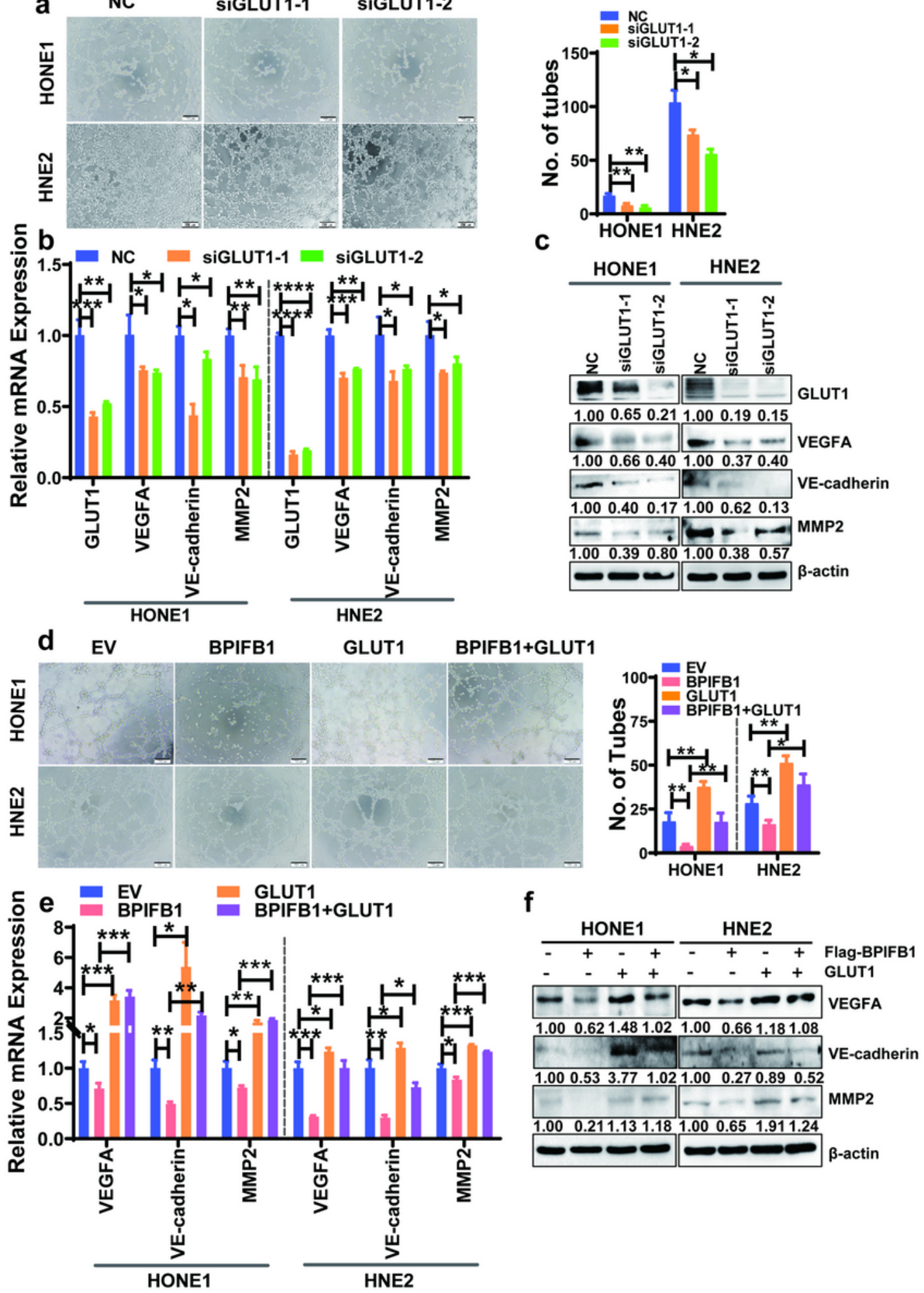

Figure 3

BPIFB1 inhibits VM formation by downregulating GLUT1. a. Tube formation assays were performed to detect tube formation by HONE1 and HNE2 cells after transfecting two GLUT1 siRNAs, respectively. b. qRT-PCR was used to examine the expression of VEGFA, VE-cadherin, and MMP2 in HONE1 and HNE2 cells after GLUT1 knockdown. c. Western blotting was used to measure the expression of VEGFA, VEcadherin, and MMP2 in HONE1 and HNE2 cells after GLUT1 knockdown. d. Tube formation assays were 
performed in HONE1 and HNE2 cells after co-transfecting the BPIFB1 and GLUT1 overexpression plasmids. e. qRT-PCR was used to examine the expression of VEGFA, VE-cadherin, and MMP2 in HONE1 and HNE2 cells after co-transfecting the BPIFB1 and GLUT1 overexpression plasmids. f. Western blotting was performed to measure the expression of VEGFA, VE-cadherin, and MMP2 in HONE1 and HNE2 cells after co-transfecting the BPIFB1 and GLUT1 overexpression plasmids. Data shown are representative images or expressed as the mean \pm s.d. of each group from three separate experiments. $\left({ }^{*}, p<0.05 ; * \star, p\right.$ $<0.01 ; * \star *, p<0.001 ; * \star * \star, p<0.0001$ vs. control, Student's t-test)

a
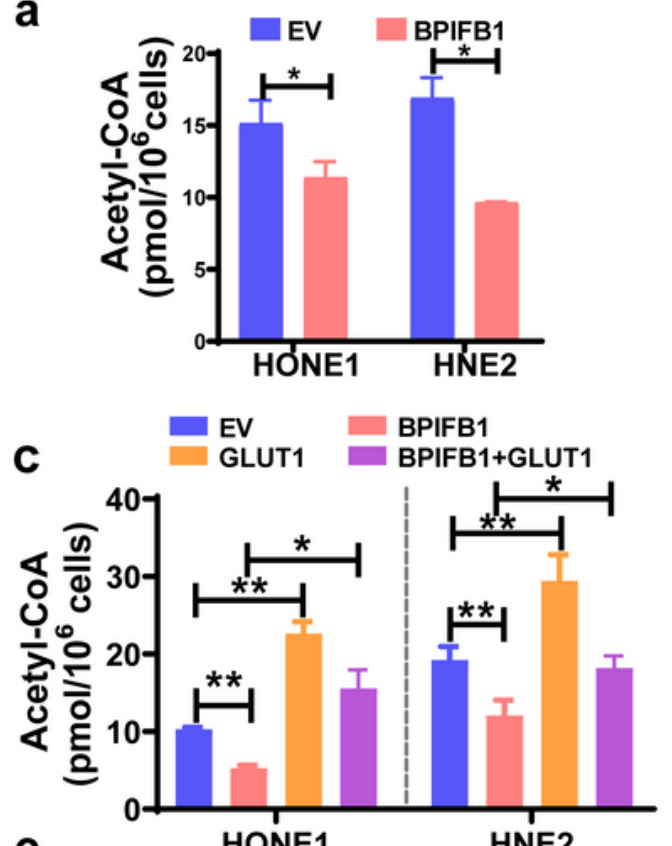

b

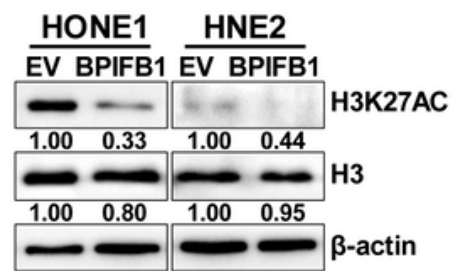

d

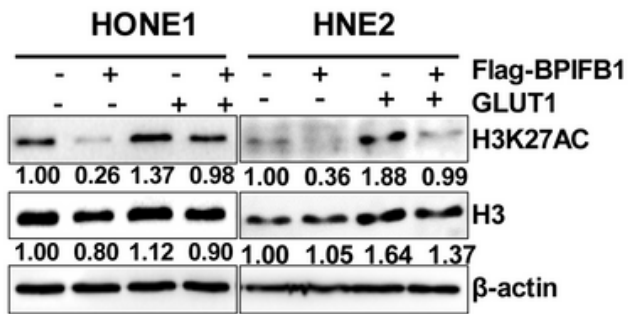

e
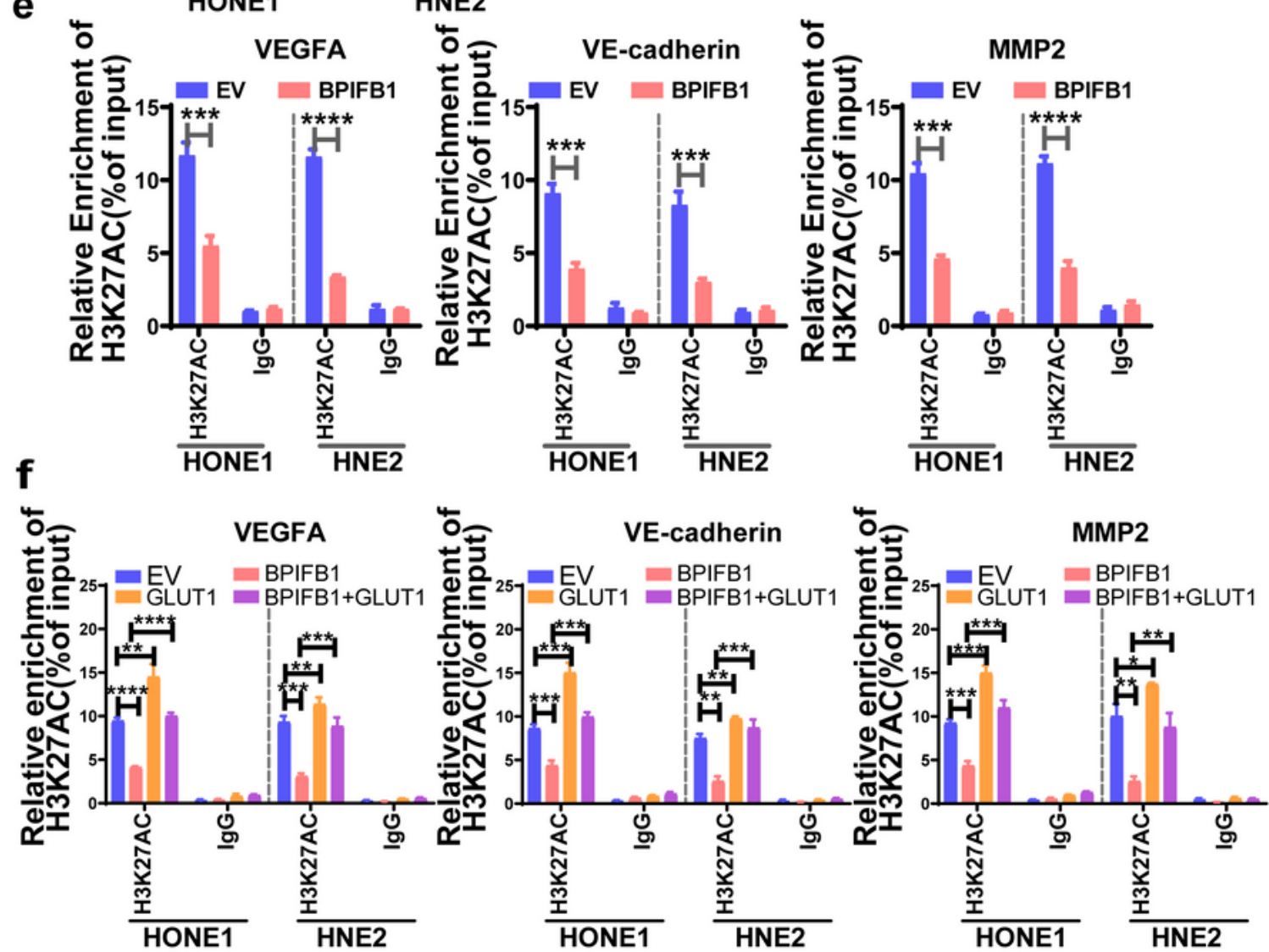


\section{Figure 4}

BPIFB1 inhibits H3K27 acetylation by downregulating GLUT1. a. The acetyl coenzyme A content in HONE1 and HNE2 cells after BPIFB1 overexpression, was measured using an acetyl coenzyme A test kit. b. H3K27AC protein expression in HONE1 and HNE2 cells after BPIFB1 overexpression was examined by western blotting. $c$. The acetyl coenzyme A content was measured in HONE1 and HNE2 cells after cotransfection of the BPIFB1 and GLUT1 overexpression plasmids, using an acetyl coenzyme A test kit. d. H3K27AC protein expression in HONE1 and HNE2 cells after co-transfection of BPIFB1 and GLUT1 overexpression plasmids was examined by western blotting. e. ChIP-qRT-PCR was used to detect H3K27AC enrichment on the VEGFA, VE-cadherin, and MMP2 genes in HONE1 and HNE2 cells after BPIFB1 overexpression. f. ChIP-qRT-PCR was used to detect H3K27AC enrichment on VEGFA, VE-cadherin, and MMP2 genes in HONE1 and HNE2 cells after co-transfection with the BPIFB1 and GLUT1 overexpression plasmids. Data shown are representative images or expressed as the mean \pm s.d. of each group from three separate experiments. ( ${ }^{*}, p<0.05$; ${ }^{* *}, p<0.01$; ${ }^{* \star}, p<0.001 ; * \star \star *, p<0.0001$ vs. control, Student's t-test) 

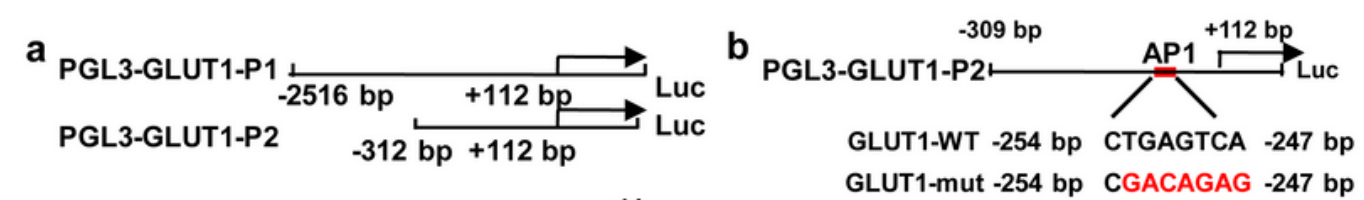
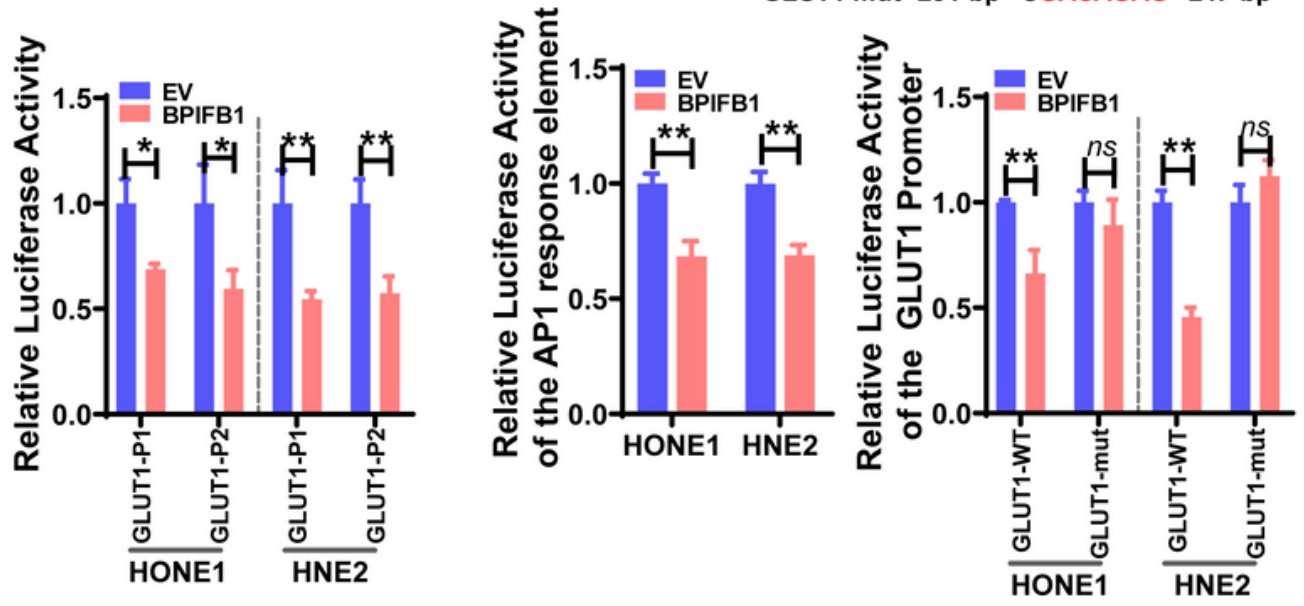

C

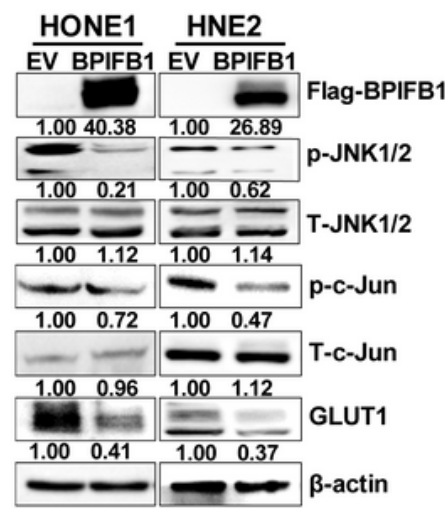

$\underset{\text { SP600125 }}{\text { d }} \frac{\text { HONE1 }}{-} \frac{\text { HNE2 }}{-}++$

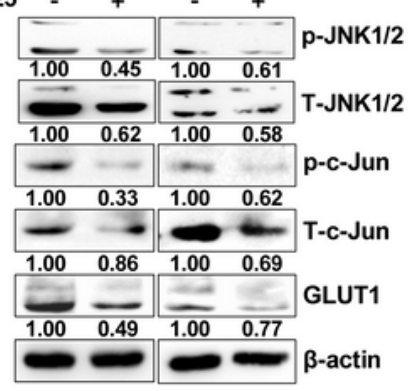

e

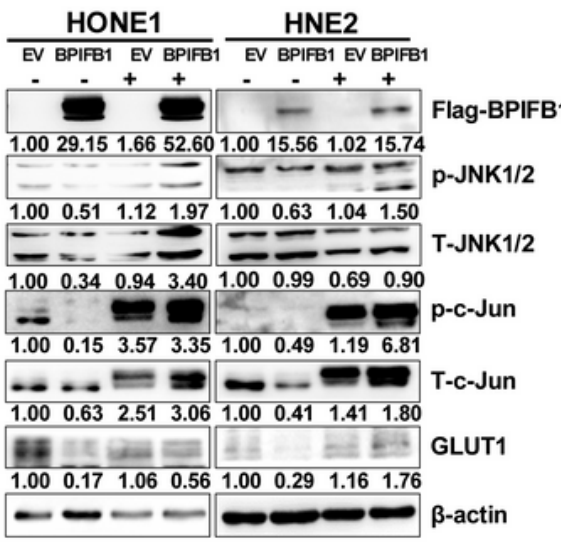

Figure 5

BPIFB1 decreases GLUT1 transcription by downregulating the JNK/AP1 pathway. a. GLUT1 promoter activity in HONE1 and HNE2 cells co-transfected with the BPIFB1 overexpression plasmid and the potential GLUT1 promoters (PGL3-GLUT1-P1 or PGL3-GLUT1-P2) was detected using a dual luciferase reporter assay. b. GLUT1 promoter activity in HONE1 and HNE2 cells co-transfected with the BPIFB1 overexpression plasmid and the wild type and mutant GLUT1 promoters (PGL3-GLUT1-P2-WT or PGL3- 
GLUT1-P2-mut) was detected using a dual luciferase reporter assay. AP1 reporter activity was used as a positive control. c. Western blotting was performed to detect the expression of p-JNK1/2, T-JNK1/2, p-cJun, T-c-Jun, and GLUT1 in HONE1 and HNE2 cells after BPIFB overexpression. d. The expression of $p-$ JNK1/2囚T-JNK1/2, p-c-Jun, T-c-Jun, and GLUT1 in HONE1 and HNE2 cells after treatment with $10 \mathrm{mM}$ SP600125 was examined by western blotting. e. The expression of p-JNK1/2, T-JNK1/2, p-c-Jun, T-c-Jun, and GLUT1 in HONE1 and HNE2 cells transfected with the BPIFB1 overexpression plasmid, after treatment with $20 \mu \mathrm{M}$ anisomycin was examined by western blotting. Data shown are representative images or expressed as the mean \pm s.d. of each group from three separate experiments. $\left({ }^{*}, p<0.05 ; * \star, p\right.$ $<0.01$ vs. control, Student's t-test) 
a

C

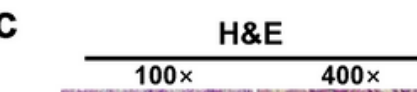

药

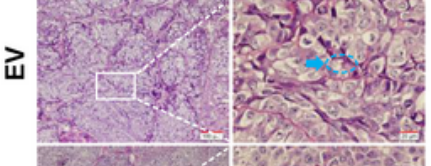

$\frac{\bar{m}}{\frac{10}{0}}$

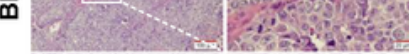

点

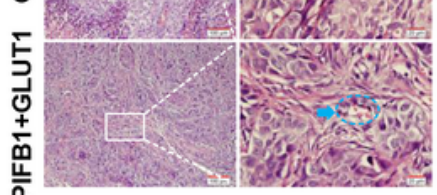

Blue Arrow:Red Cells

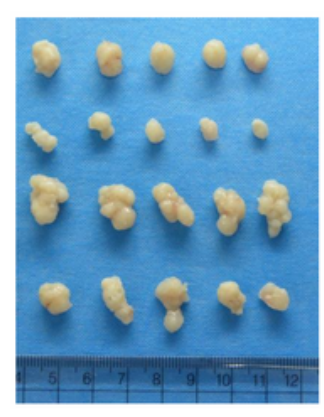

b

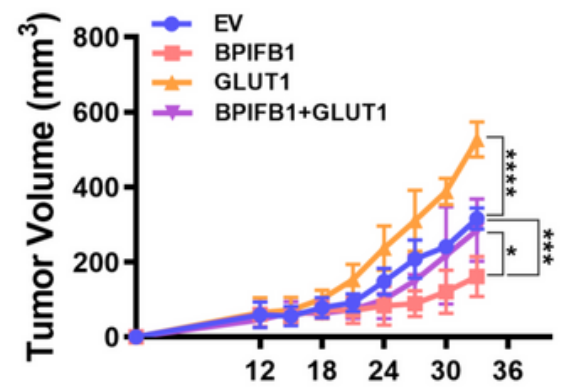

Days ${ }_{\mathrm{mCD} 31 / \mathrm{PAS}}$
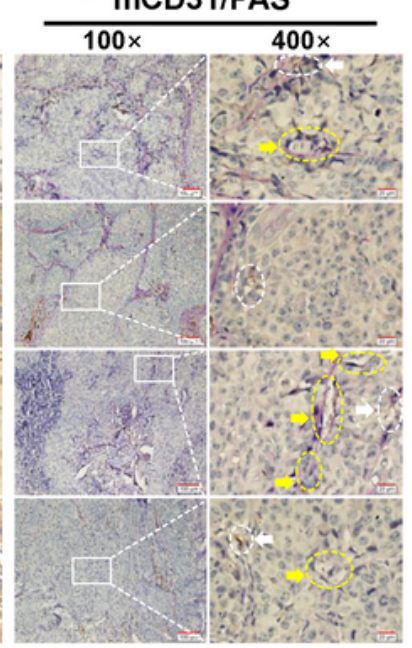

Yellow Arrow:CD31-/PAS ${ }^{+}$channel

d

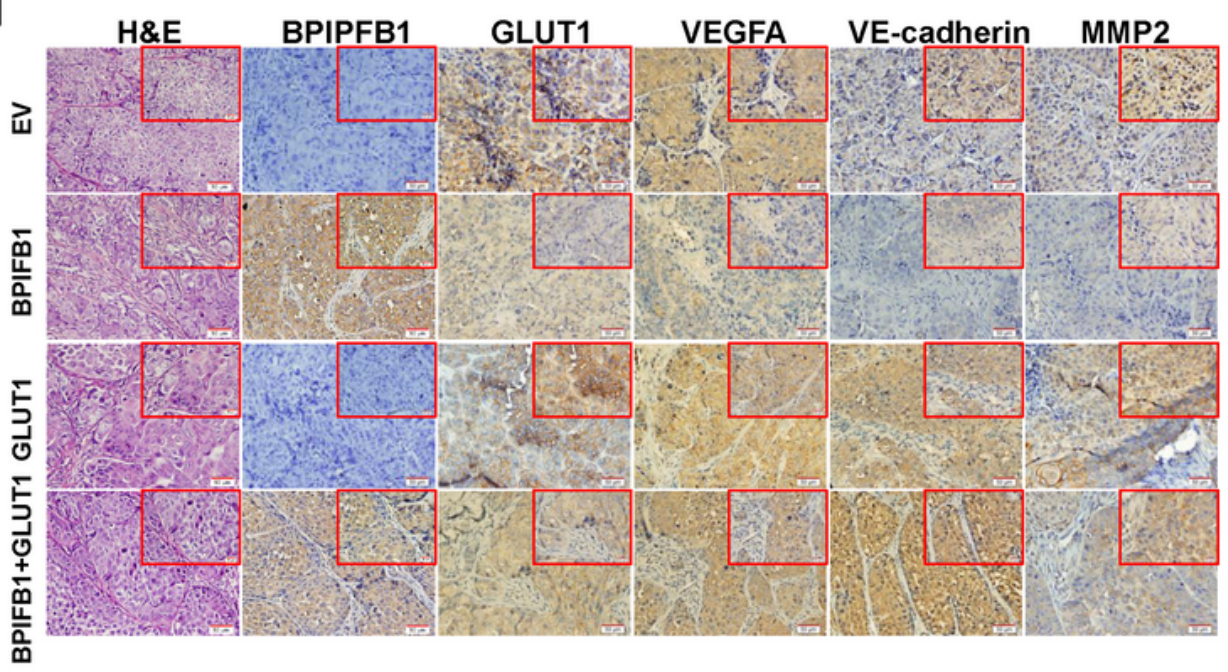

\section{Figure 6}

BPIFB1 reduces in vivo VM formation via GLUT1 downregulation. a. Pictures of tumors formed in nude mice after transplantation of HONE1 cells transfected or co-transfected with the BPIFB1 and GLUT1 overexpression plasmids. b. Statistical results of tumors formed in nude mice after transplanting HONE1 cells transfected or co-transfected with the BPIFB1 and GLUT1 overexpression plasmids. $n=5$, two-tailed Mann-Whitney test. c. H\&E staining was performed on theses tissues, BPIFB1 expression was examined 
via immunohistochemistry and VM was detected via CD31/PAS double staining in nude mice tissues. Magnification $=\times 100$, Scale bar $=100 \mu \mathrm{M}$; Magnification $=\times 400$, Scale bar $=20 \mu \mathrm{M}$. d. H\&E staining was performed on these tissues, immunohistochemistry showed the expression of BPIFB1, GLUT1, VEGFA, VEcadherin, and MMP2 in xenograft tissues. Magnification $=\times 200$, Scale bar $=50 \mu \mathrm{M}$; Magnification $=$ $\times 400$, Scale bar $=20 \mu \mathrm{M}$. Data shown are representative images or expressed as the mean \pm s.d. of each group from one separate experiment (in vivo). ( ${ }^{*}, p<0.05$; $* \star \star, p<0.001 ; * \star \star \star, p<0.0001$. vs. controls, Student's t-test or two-way ANOVA)

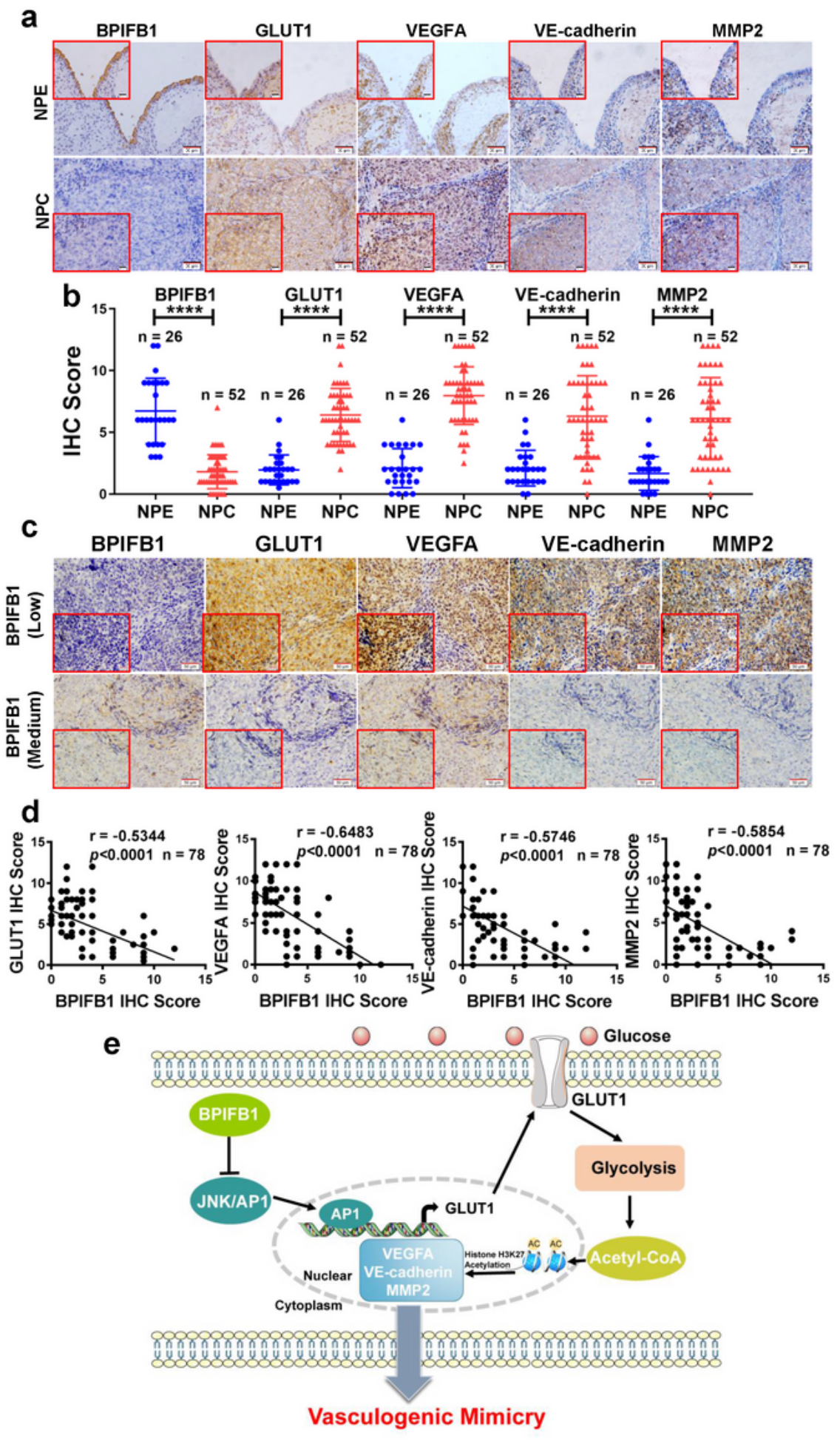




\section{Figure 7}

The relationship of BPIFB1 with GLUT1, VEGFA, VE-cadherin, and MMP2 in NPC tissues. a. Immunohistochemistry was performed to detect the expression of BPIFB1, GLUT1, VEGFA, VE-cadherin, and MMP2 in 26 NPE tissues and 52 NPC tissues. Magnification $=\times 200$, Scale bar $=50 \mu$; Magnification $=\times 400$, Scale bar $=20 \mu \mathrm{M}$. b. The statistical results of BPIFB1, GLUT1, VEGFA, VE-cadherin, and MMP2 expression in 26 NPE and 52 NPC tissues. c. Representative images showing the correlation between BPIFB1 and GLUT1, VEGFA, VE-cadherin, and MMP2 expression in NPC tissues. Magnification = $\times 200$, Scale bar $=50 \mu \mathrm{M}$; Magnification $=\times 400$, Scale bar $=20 \mu \mathrm{M}$. d. The statistical results showed correlation of BPIFB1 with GLUT1, VEGFA, VE-cadherin, and MMP2 expression in NPC tissues. e. Model for inhibition of VM formation by BPIFB1 through downregulation of GLUT1-mediated H3K27 acetylation. Data shown are representative images or expressed as the mean \pm s.d. of each group from one separate experiment (in vivo), ( ${ }^{\star * \star *}, p<0.0001$. Student's t-test)

\section{Supplementary Files}

This is a list of supplementary files associated with this preprint. Click to download.

- SupplementalTable1.pdf

- SupplementalTable2.pdf

- SupplementalTable3.xlsx

- SupplementalTable4.pdf

- Supplementary.docx 